\title{
JEFFRIES MATUSITA-SPECTRAL ANGLE MAPPER (JM-SAM) SPECTRAL MATCHING FOR SPECIES LEVEL MAPPING AT BHITARKANIKA, MUTHUPET AND PICHAVARAM MANGROVES
}

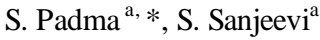 \\ ${ }^{a}$ Remote Sensing Unit, Department of Geology, Anna University, India - padmagi91@ gmail.com, ssanjeevi@annauniv.edu
}

\author{
Commission VIII
}

KEY WORDS : Spectral Matching, hyperspectral image, Jeffries-Matusita, Spectral Angle Mapper, classification

\begin{abstract}
ABS TRACT:
This paper proposes a novel hyperspectral matching algorithm by integrating the stochastic Jeffries-Matusita measure (JM) and the deterministic Spectral Angle Mapper (SAM), to accurately map the species and the associated landcover types of the mangroves of east coast of India using hyperspectral satellite images. The JM-SAM algorithm signifies the combination of a qualitative distance measure (JM) and a quantitative angle measure (SAM). The spectral capabilities of both the measures are orthogonally projected using the tangent and sine functions to result in the combined algorithm. The developed JM-SAM algorithm is implemented to discriminate the mangrove species and the landcover classes of Pichavaram (Tamil Nadu), Muthupet (Tamil Nadu) and Bhitarkanika (Odisha) mangrove forests along the Eastern Indian coast using the Hyperion image dat asets that contain 242 bands. The developed algorithm is extended in a supervised framework for accurate classification of the Hyperion image. The pixel-level matching performance of the developed algorithm is assessed by the Relative Spectral Discriminatory Probability (RSDPB) and Relative Spectral Discriminatory Entropy (RSDE) measures. From the values of RSDPB and RSDE, it is inferred that hybrid JM-SAM matching measure results in improved discriminability of the mangrove species and the associated landcover types than the individual SAM and JM algorithms. This performance is reflected in the classification accuracies of species and landcover map of Pichavaram mangrove ecosystem. Thus, the JM-SAM (TAN) matching algorithm yielded an accuracy better than SAM and JM measures at an average difference of $13.49 \%, 7.21 \%$ respectively, followed by JM-SAM (SIN) at $12.06 \%, 5.78 \%$ respectively. Similarly, in the case of Muthupet, JM-SAM (TAN) yielded an increased accuracy than SAM and JM measures at an average difference of $12.5 \%$, $9.72 \%$ respectively, followed by JM-SAM (SIN) at $8.34 \%, 5.55 \%$ respectively. For Bhitarkanika, the combined JM-SAM (TAN) and (SIN) measures improved the performance of individual SAM by $(16.1 \%, 15 \%)$ and of JM by $(10.3 \%, 9.2 \%)$ respectively.
\end{abstract}

\section{INTRODUCTION}

\subsection{Introduction}

The quality of remote sensing applications has increased significantly with the availability of abundant spectral information. Spectral research is the detailed analysis and interpretation of the spectra of materials. In recent times, spectral matching algorithms have been developed to adapt and extract information from the real-time hyperspectral datasets. Though several spectral matching approaches were developed to increase the information extraction from hyperspectral data, each of these methods has its own limitation in utilizing the band-level information and their performances is not of acceptable quality. It was also inferred by many authors that combining the spectral abilities of two or three matching algorithms will overcome the limitations present in the individual algorithms. Further the spectral separability measures namely Jeffries-Matusita distance has only been used in the context of image classification and not in spectral matching. Hence, the aim of this research is to develop a novel matching algorithm that incorporate signature separability measure such as Jeffries-Matusita distance along with the deterministic Spectral Angle Mapper (SAM), for improved classification of mangrove ecosystems using hy perspectral datasets.

\subsection{Spectral matching and mangrove mapping}

Mangroves are salt-tolerant species located in the tropical and subtropical regions and form a complex ecosystem along the Indian coasts. In the Indian context, the east coastline comprises of major mangrove ecosystems such as Sunderbans (West Bengal), Bhitarkanika (Odisha), Coringa (Andhra Pradesh), Pichavaram and Muthupet (Tamil Nadu). These forests contribute towards the $4 \%$ of global mangrove population. Further, these ecosystems influence the floral diversity, landuse,

\footnotetext{
* Corresponding author.

Email address: padmagi91@gmail.com (S. Padma)
} 
population, vegetation prospects and livelihood aspects of the surrounding regions. While several ground-based techniques were used to evaluate and manage the mangrove ecosystems, the advent of remote sensing technology paved way for an effective and potential method of mangrove mapping. Several studies of the past (Selvam et al., 2003, 2010; Ajithkumar et al., 2008) stated the difficulty to map the complex mangrove ecosystems at species level using the multispectral datasets. With the availability of hyperspectral datasets, the possibility to utilize the spectral characteristics of the species in identifying is realized in the previous researches on species mapping. Further, spectral matching using hyperspectral datasets enables the complete incorporation of the spectral information for classification and precise identification of mangrove ecosystem.

\section{STUDY S ITE CHARACTERIS TICS}

\subsection{Hyperspectral image data used}

EO-1 Hyperion image datasets (shown in Figure 2 (a), b and c) is used for the demonstration of the spectral matching based classification. The sensor parameters of the image scenes used in this study are given in Table 1. Out of 242 bands, only 198 bands are calibrated due to low response of the detector (Beck 2003).

\begin{tabular}{|c|c|}
\hline Image characteristics & EO-1 Hyperion \\
\hline Spectral Range & $0.4-2.5 \mu \mathrm{m}$ \\
\hline Spatial Resolution & $30 \mathrm{~m}$ \\
\hline Radiometric Resolution & $16 \mathrm{bits} / \mathrm{pixel}$ \\
\hline Swath Width & $7.5 \mathrm{~km}$ \\
\hline Spectral Resolution & $10 \mathrm{~nm}$ \\
\hline Spectral Coverage & Continuous \\
\hline Date of Acquisition & $\begin{array}{c}\text { January 03, 2013 (Pichavaram) } \\
\text { October 07, 2007 (Muthupet) } \\
\text { January 14, 2008 (Bhitarkanika) }\end{array}$ \\
\hline
\end{tabular}

Table 1. Specifications of the hyperspectral images used

\subsection{Study Sites}

The spectral matching algorithms were used to characterize the Pichavaram, Muthupet and Bhitarkanika mangrove ecosystems found along the Eastern coast of India.

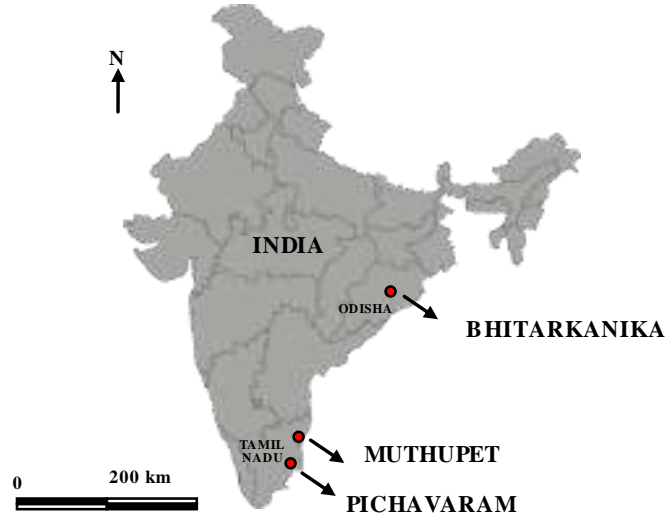

Figure 1. Map showing the location of Pichavaram and Muthupet and Bhitarkanika, East coast India

Pichavaram mangrove ecosystem located in Chidambaram, Cuddalore District of southern India comprise of 13 species of mangroves. Pichavaram is dominated by these two species namely Avicennia and Rhizophara which constitute around $89 \%$ of the total population of mangroves. The climate is sub-humid with maximum rainfall during the northeast monsoons (Selvam et al., 2003). The Rhizopora zone, as a narrow strip along the tidal creeks and channels occurs at breadth of $4 \mathrm{~m}$, while the Avicennia zone is of $90 \mathrm{~m}$. This phenomenon of Rhizopora species (dark green in color) bordering the entire Avicennia species (light green in color) can be seen in Figure 2 (a). Groundnut and paddy are the major crops cultivated in Pichavaram (Selvam et al., 2002).

Muthupet mangroves forest is located in the southernmost end of the Cauvery delta, Tiruvarur District of southern India. around 6 mangroves species where the Avicennia occupies around $95 \%$ of the total population. The Avicennia zone occurs in the fringe area of the tidal creeks with dense evergreen trees of 3-8 $\mathrm{m}$ heights and its breadth varies from a few meters in the fringe to $2.5 \mathrm{~km}$. Besides, large tracts of Prosopis juliflora, mudflats and other plantations are found all along the landward margin.

Bhitarkanika Wildlife Sanctuary is a rich and lush green ecosystem lying in north-eastern corner of Kendrapara district of Odisha on east coast of India. Bhitarkanika wetland, considered as one of the important mangrove genetic resources of the world, was declared a Ramsar site in the year 2002. The area has 372 species of flowering plants including 58 species of mangrove

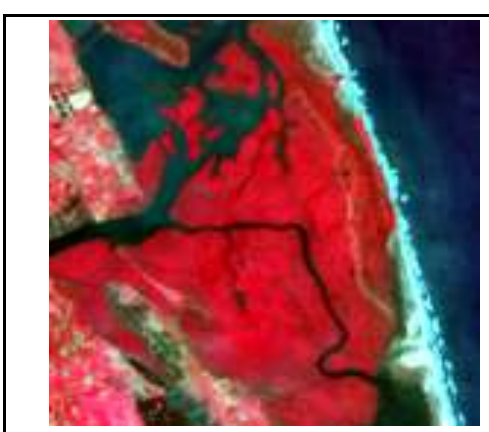

(a)

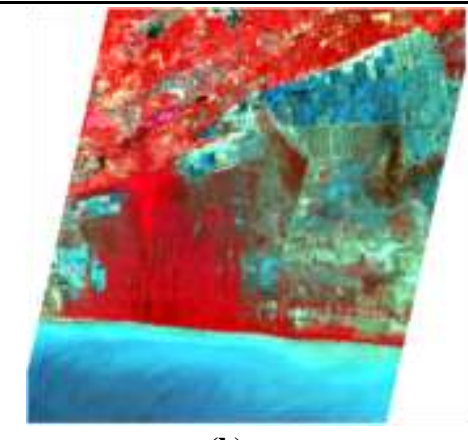

(b)

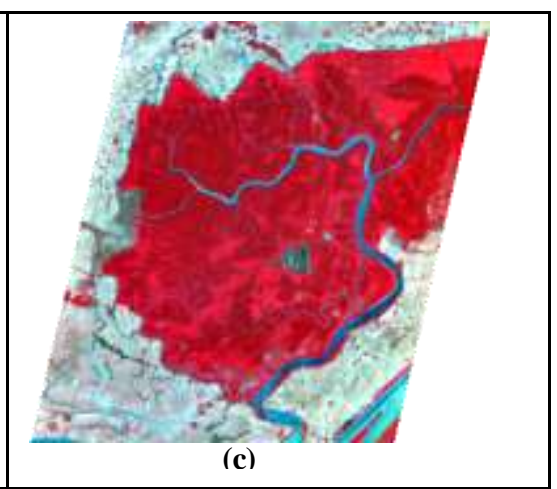

Figure 2. EO-1 Hy perion image of (a) Pichavaram, (b) Muthupet. and (c) Bhitarkanika FCC (R=B40, G=B30, B=B20) 
species. Heritiera kanikensis, is endemic and a dominant mangrove species in the Bhitarkanika mangrove wetland. In addition, two other mangrove species namely Excocaria and Avicennia are found in combination in some parts. The associated landuse includes the casuarina plantation and mudflats.

\section{METHODOLOGY}

The workflow involves of developing the JM-SAM algorithm, followed by the implementation of the algorithm for improved landcover classification using hyperspectral datasets in the MATLAB environment. After a detailed pre-processing, the reference spectral signatures (Figure 3) are extracted from the hyperspectral datasets. These signatures are assessed for nonnormality which is a pre-requisite for implementing the JM measure. Then, the pixel based analysis and matching based classification is carried out using the JM, SAM and the developed JM-SAM combined algorithm. The performance of the JM-SAM algorithm compared to the SAM and JM measures is evaluated based on the values of relative spectral discriminatory probability (RSDPB) and the relative spectral discriminatory entropy (RSDE). Further the post-classification accuracy assessment is carried out and related with the RSDPB and RSDE measures to substantiate the performance of the matching algorithms. Along with a species map, Google Earth image is used as reference to assess the performance of the classifier. The effectiveness of the algorithm is compared to the SAM and JM measures based on the classification accuracy. Further, the results are compared with the (standard) Minimumdistance classifier.

\section{JM-S AM COMBINED ALGORITHM}

Spectral Angle Mapper is a measure of the spectral angle between the target spectrum and the reference spectra (Kruse et al., 1993). A smaller angle is seen for higher spectral similarity. $\operatorname{SAM}(\theta)$ for spectra $S_{1} \& S_{2}$ along wavelength $\lambda$ is:

$$
\theta=\cos ^{-1}\left[\frac{\int \mathrm{s}_{1}(\lambda) \mathrm{S}_{2}(\lambda) \mathrm{d} \lambda}{\left[\int \mathrm{S}_{1}(\lambda) \mathrm{d} \lambda\right]^{\frac{1}{2}}\left[\int \mathrm{s}_{2}(\lambda)\right]}\right]
$$

Though SAM captures the intrinsic properties of materials in terms of spectral angle (Vishnu et al., 2013), it is insensitive to illumination effects. Hence, SAM has difficulty in identifying spectrally similar materials. Several improvisations have been made to the basic SAM measure, and it is used in combination with the stochastic divergence measures (Du et al., 2004).

Jeffries-Matusita distance is one of the spectral separability measures commonly used in remote sensing applications. According to Swain et al (1973), JM distance provides a much reliable criterion because as a function of separability, it behaves much more like probability of correct classification. The probability density of the spectral vectors, $S_{1}$ and $S_{2}$ for the bands $(\mathrm{l}=1,2 \ldots \mathrm{L})$ is $\mathrm{p}_{1}$ and $\mathrm{q}_{1}$ and the JM distance (Swain et al., 1973; Chang, 2003 and Ghiy amat et al., 2013) is given as:

$$
\mathrm{JM}\left(\mathrm{S}_{1}, \mathrm{~S}_{2}\right)=\sqrt{\sum_{l=1}^{L}\left[\sqrt{p_{l}}-\sqrt{q_{l}}\right]^{2}}
$$

In the present study, the spectral vectors chosen for matching are distributed non-normally. Hence the basic equation may not result in accurate matching (Ghiy amat et al., 2013). JM distance (Equation. 3.1) is used for the non-normally distributed spectral densities (Chang, 2003 and Ghiy amat et al., 2013). Here the JM distance is similar to the Spectral Information Divergence (SID) measure as it quantifies the band-wise information between the spectral vectors (Chang, 2003).

The proposed algorithm (JM-SAM) is developed by combining the deterministic Spectral Angle Mapper (SAM) (Equation 1) and the stochastic Jeffries-Matusita Measure (JM) (Equation 2) by the tangent and sine functions. The JM-SAM algorithm is measured as:

$\operatorname{JM}-\operatorname{SAM}(\mathrm{TAN})=\operatorname{JM}\left(\mathrm{S}_{1}, \mathrm{~S}_{2}\right) \times \tan \left(\operatorname{SAM}\left(\mathrm{S}_{1}, \mathrm{~S}_{2}\right)\right)$

$\operatorname{JM}-\operatorname{SAM}(\operatorname{SIN})=\operatorname{SID}\left(\mathrm{S}_{1}, \mathrm{~S}_{2}\right) \times \sin \left(\operatorname{SAM}\left(\mathrm{S}_{1}, \mathrm{~S}_{2}\right)\right)$

The tangent and sine trigonometric functions are used to calculate the perpendicular distance between the target and reference $\left(S_{1}\right.$ and $\left.S_{2}\right)$ respectively, instead of the cosine function which projects one spectrum along the other (Du et al., 2004 and Naresh Kumar et al., 2011).

\section{IMPLEMENTATION OF S PECTRAL MATCHING BASED CLASS IFICATION}

The developed matching algorithm is extended in a supervised framework for improved classification of the Hyperion image. The reference spectra of the mangrove species and other associated cover types are extracted from the Hyperion images which were corrected for atmospheric errors. From the Hyperion image of Pichavaram, reference spectra for Avicennia and Rhizopora species, paddy, groundnut, mudflat, sand, clear and turbid water were obtained. In the case of Muthupet region, spectra pertaining to dense and sparse Avicennia species, Prosopis, plantation, mudflat, sand, clear and turbid water are extracted. Similarly for Bhitarkanika, reference spectra are extracted for Heritiera (fully grown), Heritiera (partially grown), Excoecaria, mixed (Excocaria-Avicennia), agriculture, mudflat, barren land, clear and turbid water. These spectra are assessed for non-normality distribution, which is a pre-requisite for the implementation of the developed matching algorithm. These spectra are collected and stored in the form a repository or database called the Spectral Library.

Each spectrum in the library is considered as a reference candidate for matching. In this spectral matching-based classification approach, each pixel in the study area is considered as a target spectrum. The matching values between the reference spectrum in the library and target spectrum in the image are obtained using the combined measures JM-SAM (TAN), JM-SAM (SIN), and the individual measures such as JM and SAM. The target is labelled to the corresponding reference pixel based on the least matching value. This process results in the classified images (Figure 4). 


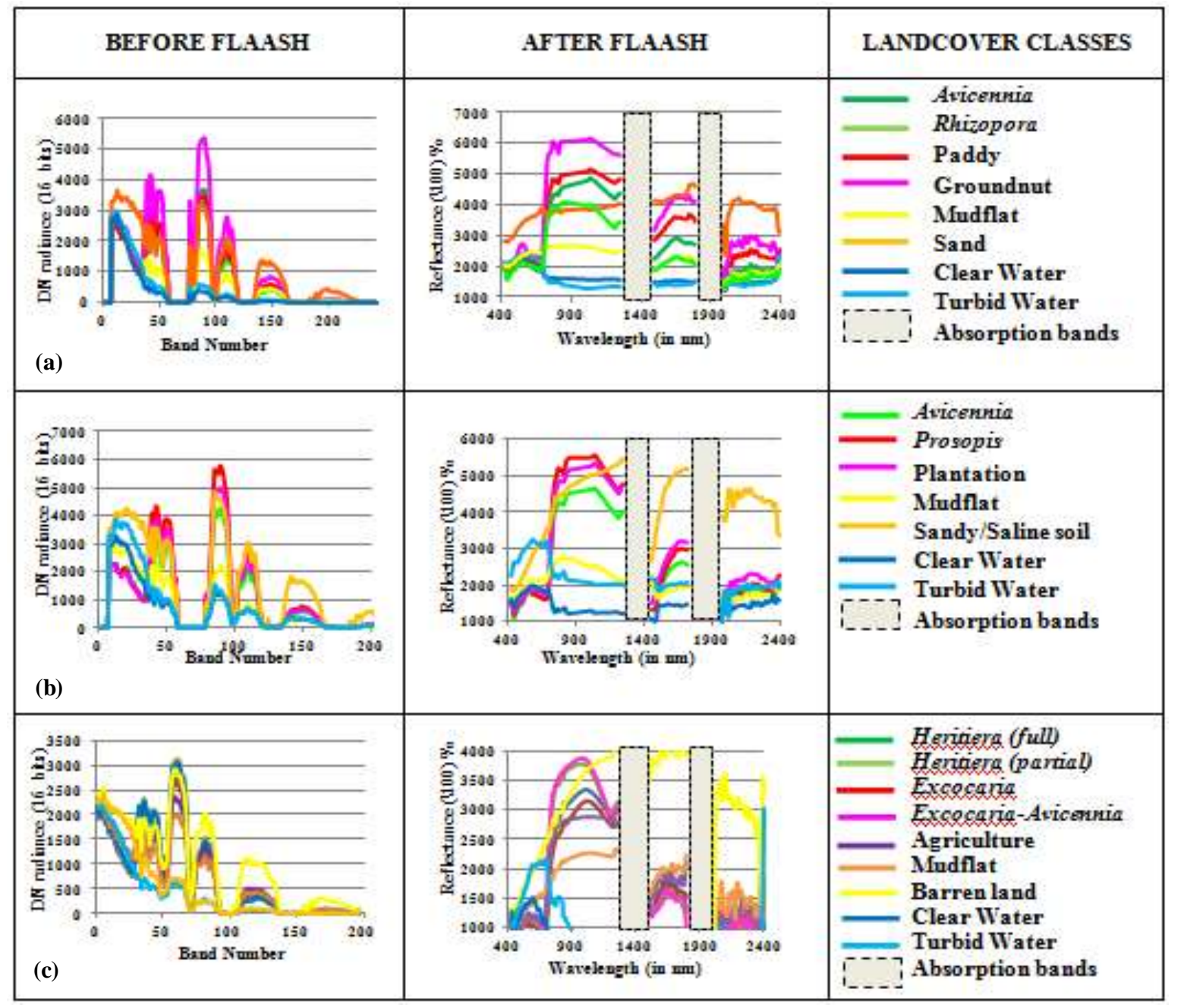

Figure 3. Illustration of the reference spectra of landcover types obtained before and after FLAASH from (a) Pichavaram (b) Muthupet and (c) Bhitarkanika using the EO-1 Hyperion image data.

\section{EVALUATION MEASURES}

The pixel-based matching performances of the algorithms are evaluated by the RSDPB and RSDE measures. The postclassification accuracy assessment is carried out to evaluate the correctness in labelling of each class in the image. Further, trendlines are generated by relating the entropy (RSDE) measures and classification accuracy values.

\subsection{Relative Spectral Discriminatory Probability (RSDPB)}

The relative spectral discriminatory probability (RSDPB) (Chang, 2003; Du et al., 2004 and Dudeni et al., 2009), is the measure of likelihood of the identification of the target signature from a set of spectral signatures or spectral library. It is the normalized distance between the target and the respective reference spectra. From the resulting RSDPB vector, the reference unit with a least relative probability is assumed as the best match for the target.

\subsection{Relative Spectral Discriminatory Entropy (RSDE)}

Using the RSDPB vector, the RSDE (Chang, 2003; Du et al., 2004 and Dudeni et al., 2009) is computed to measure the uncertainty of matching the target spectra with the reference spectra in the spectral library. It is to be noted that, larger the value of RSDE, smaller is the chance of identifying a target from the set of reference spectra in the library.

\subsection{Accuracy Assessment}

In order to estimate the percentage of correctly classified or matched pixels, the post classification accuracy assessment is carried out. Here, around 160 pixels in the classified image are selected through a stratified random process for accuracy estimation using the ERDAS Imagine package. Further these pixels were validated by identifying their respective landcover samples using Google Earth (Geo-Eye-I) image. In the case of Pichavaram and Muthupet, the species composition maps prepared by the MSSRF (Selvam et al., 2003 and 2010), India were also referred for validation. For Bhitarkanika region, a community map prepared by Reddy et al., 2008 was referred. 


\section{RESULTS AND DISCUSSION}

\subsection{Matching-based classification of Pichavaram image}

The degree of match for the Hyperion image can be assessed by the nature of discrimination done for the spectrally similar targets: Avicennia, Rhizopora, paddy and groundnut. In assessing the spectral similarity of Avicennia with Rhizopora, paddy and groundnut, the matching scores produced by the JM SAM combined measures are $0.0029,0.0033$ and 0.0055 respectively. For the same scenario, SAM and JM produce similarity values of $0.0739,0.0789,0.0963$ and $0.0387,0.0417$ and 0.0567 respectively. JM-SAM low values of similarity compared to the individual SAM and JM measures indicate the increased capability of the combined algorithm to discriminate even closely matching spectra. This is due to a combination of the least separable distance and the spectral angle between the reference and target vector.

Better performance of the JM-SAM algorithm compared to the individual measures of SAM and JM was observed from the RSDPB values. Similarity and dissimilarity between the target and reference spectra are captured efficiently by the JM-SAM.

At a difference of 0.034 and 0.033 (in the range of 0-0.3), the JM-SAM (TAN) and JM-SAM (SIN) measures shows improved performance than the SAM approach in discriminating Avicennia from Rhizopora. Similarly, a difference of 0.037 and 0.036 in the range of $0-0.3$ indicates the improved performance of JM-SAM (TAN) and JM-SAM (SIN) measures than the JM approach. The improved performance of JM-SAM algorithm is attributed to its nature of combining the least separable distance between the Avicennia and Rhizopora spectra at each band, along with the spectral angle between them. Such discrimination is reflected in the classification results where the JM-SAM (TAN) and JM-SAM (SIN) resulted in accuracy of $(90.5 \%, 75.5 \%)$ and $(91.5 \%, 78.5 \%)$ in labeling Avicennia and Rhizopora.

The RSDE measures for the eight spectral classes also indicate the discriminatory ability of JM-SAM, which has lowest relative entropy compared to the SAM and JM measures. The least average entropy measure, which indicates the higher chance of target matching, is obtained through JM-SAM (TAN) at a value of 2.39 , followed by 2.37 of JM-SAM (SIN). The SAM approach has the highest average entropy value of 2.61 followed by 2.65 of JM. The lower uncertainty of JM-SAM algorithm in identifying the correct match is the reason to its better performance.

It is seen that the classification accuracy increases with decreasing range of RSDE measure (Figure 5). For instance, the JM-SAM (TAN) measure classifies paddy at an accuracy of $92.5 \%$ at a RSDE of 2.07 , while the JM measures classify at 42.5 at a RSDE of 2.53. This improved performance of the JMSAM measure is inferred for all the eight classes at a lower range of entropy measure.
Besides, the JM-SAM spectral matching approach presented here results in improved accuracy compared to the minimum distance approach (Table 2). The order of classification accuracy is as follows: JM-SAM $>\mathrm{JM}>\mathrm{SAM}>\mathrm{M}$ inimum Distance approach.

\begin{tabular}{|c|c|c|c|c|c|}
\hline \multirow{3}{*}{ Class } & \multicolumn{5}{|c|}{ Accuracy of classification (in \%) } \\
\hline & \multirow[t]{2}{*}{ MIN } & \multirow[t]{2}{*}{ SAM } & \multirow{2}{*}{$\mathbf{J M}$} & \multicolumn{2}{|c|}{ JM-SAM } \\
\hline & & & & TAN & SIN \\
\hline$A$ & 56.2 & 71 & 56.2 & 90.5 & 91.5 \\
\hline$R$ & 78.1 & 73.2 & 78.1 & 75.5 & 78.5 \\
\hline $\mathrm{P}$ & 40.5 & 80.5 & 40.5 & 92.5 & 78.5 \\
\hline $\mathrm{G}$ & 40.8 & 32.5 & 40.8 & 52.5 & 68.5 \\
\hline M & 70.5 & 62.5 & 70.5 & 100 & 100 \\
\hline $\mathrm{S}$ & 81.5 & 90.3 & 81.5 & 100 & 100 \\
\hline C & 80 & 91.5 & 80 & 90.5 & 90.8 \\
\hline $\mathrm{T}$ & 98 & 83 & 98 & 100 & 100 \\
\hline $\mathrm{OA}$ & 67.50 & 71.25 & 67.50 & 86.25 & 85 \\
\hline OK & 0.63 & 0.67 & 0.63 & 0.84 & 0.83 \\
\hline \multicolumn{6}{|c|}{$\begin{array}{l}\text { Note: A-Avicennia, } R \text {-Rhizopora, P-Paddy, G-Groundnut, } \\
\text { M-Mudflat, S-Sand, C-Clear water, T-Turbid water, OA- } \\
\text { Overall accuracy, OK - Overall Kappa }\end{array}$} \\
\hline
\end{tabular}

Table 2. Results of Accuracy Assessment for classification of Pichavaram image

\subsection{Matching -based classification of Muthupet image}

The consistent performance of JM-SAM in classification is further confirmed with the spectral matching values of the seven classes extracted from the Hyperion image for Muthupet mangrove ecosystem. The order of matching values is as follows: SAM > JM > JM-SAM, indicating the increased matching of JM-SAM which has the least value.

The matching ability of JM-SAM for the Muthupet dataset can be assessed by its nature of discrimination for the spectrally similar targets: clear water and turbid water. The JM-SAM combined measures produced values of 0.0127 and 0.0128 while the SAM and JM produced values such as 0.1527 and 0.0833 in matching clear water with turbid water. The lower values of the combined measure indicate the range of discriminability, it adopted for identifying the differences between closely matching spectra.

Further, the better performance of the JM-SAM algorithm compared to the individual measures of SAM and JM was observed from the RSDPB values. At a RSDP difference of 0.0159 and 0.0187 in the range of (0-1), the JM-SAM (TAN) and At a difference of 0.041 and 0.045 (in the range of $0-0.1$ ), the JM-SAM (TAN) indicated an improved performance than SAM and JM measures in discriminating clear water from turbid water. In the same scenario, JM-SAM (SIN) displays increased performance than the JM and SAM approach with a RSDPB 
difference of 0.038 and 0.042 in the range of $0-1$. This improved performance of JM-SAM is attributed to its nature of combining the least separable distance between the clear water and turbid water spectra at each band, along with the spectral angle between them. This discrimination is reflected in the classification results where the JM-SAM (TAN) and JM-SAM (SIN) show an accuracy of $(83.31 \%, 100 \%)$ and $(85.71 \%, 100 \%)$ in identifying clear water and turbid water.

The RSDE values for the seven spectral classes also indicate the discriminability of JM-SAM measures, which has lowest relative entropy $(2.25,2.23)$ compared to the SAM (2.42) and JM (2.44) measures. For instance, the JM-SAM (TAN) and JM-SAM(SIN) measures classifies Avicennia at an accuracy of $100 \%$ at a RSDE of 1.93 and 1.97 respectively, while SAM and JM measures classify at $71.43 \%$ at a RSDE of 2.29 and 2.32 respectively. This improved performance of JM-SAM measure is observed for all the seven classes at a lower range of entropy measure. From the trendlines (Figure 6) relating the RSDE values with the classification accuracy measures, it can be inferred that for all the seven spectral classes, JM-SAM has the lowest relative entropy and highest classification accuracy compared to the SAM and JM measures.

\begin{tabular}{|c|c|c|c|c|c|}
\hline \multirow{3}{*}{ Class } & \multicolumn{5}{|c|}{ Accuracy of classification (in \%) } \\
\hline & \multirow[t]{2}{*}{ MIN } & \multirow[t]{2}{*}{ SAM } & \multirow[t]{2}{*}{$\mathbf{J M}$} & \multicolumn{2}{|c|}{ JM-SAM } \\
\hline & & & & TAN & SIN \\
\hline$A$ & 60 & 71.43 & 71.43 & 100 & 100 \\
\hline$P$ & 70 & 75.20 & 71.43 & 66.67 & 75 \\
\hline PL & 70 & 33.33 & 85.71 & 83.33 & 71.43 \\
\hline M & 80 & 66.67 & 71.43 & 83.33 & 85.71 \\
\hline$S$ & 50 & 62.50 & 85.71 & 100 & 85.71 \\
\hline $\mathrm{C}$ & 80 & 82.35 & 71.43 & 83.31 & 85.71 \\
\hline $\mathrm{T}$ & 60 & 92.31 & 85.71 & 100 & 100 \\
\hline $\mathrm{OA}$ & 67.14 & 71.43 & 77.55 & 88.10 & 86.96 \\
\hline $\mathrm{OK}$ & 0.62 & 0.65 & 0.74 & 0.86 & 0.85 \\
\hline \multicolumn{6}{|c|}{$\begin{array}{l}\text { Note: A-Avicennia, P-Prosopis, PL-Plantation, M-Mudflat, } \\
\text { S-Saline/Sandy soil, C-Clear water, T-Turbid water, OA- } \\
\text { Overall Accuracy, OK-Overall Kappa }\end{array}$} \\
\hline
\end{tabular}

Table 3. Results of Accuracy Assessment for classification of Muthupet image

Further, the JM-SAM spectral matching approach presented provides an improved accuracy than the minimum distance approach (Table 3). The order of classification accuracy is as follows: JM-SAM > JM > SAM > Minimum Distance approach.

\subsection{Matching-based classification of Bhitarkanika image}

In the case of Bhitarkanika, spectrally similar targets namely Heritiera (fully grown) and Heritiera (partially grown) are captured effectively by the JM-SAM combined algorithm. The spectral differences existing between Heritiera (fully grown) and Heritiera (partially grown) are evaluated by a score of 0.0020 and 0.0019 by the JM-SAM (TAN) and (SIN) algorithms. On the other hand, the SAM and JM measures produced similarity values of 0.075 and 0.070 . The lower values of the JM-SAM measure confirms the improved capability of the combined algorithm to discriminated closely matching spectra that occurs within the same type of species at different stages of their growth. Similarly, the mixed mangrove spectrum comprising of Excocaria and Avicennia were discriminated from the individual Exocaria by the JM-SAM (TAN) measure at a difference of 0.072 and 0.065 compared to the SAM and JM measures. In the case of JM-SAM (SIN), a difference of 0.070 and 0.063 existed compared to SAM and JM measures.

The RSDPB performance values for JM-SAM measures further confirmed the improved performance in identifying classes having closely similar spectra. At a difference of 0.030 and 0.029 in the range of $0-0.3$, JM-SAM (TAN) and JM-SAM (SIN) measures improved the matching performance compared to SAM approach in discriminating Heritiera (fully grown) from the partially grown variety. In the same scenario, at a difference of 0.032 and 0.029 in the range of 0-0.3, the combined measures outperformed the JM measure. This nature of JM-SAM measures to utilize the least spectral angle and least spectral between Heritiera (fully grown) and (partially grown) variety along each band was reflected in the classification results. The JM-SAM (TAN) and JM-SAM (SIN) measures yielded an increased accuracy of $(94 \%, 92.5 \%)$ and $(93.5 \%, 93 \%)$ in labelling the Heritiera varieties.

The RSDE measures indicate that the uncertainty level in identifying the correct matches is least in the case of JM-SAM approaches compared to the SAM and JM. The least average entropy measure in the case of JM-SAM (TAN) is 2.35, followed by 2.33 of JM-SAM (SIN). The highest entropy value indicating the increased uncertainty in accurate matching was that of SAM (2.73) followed by JM (2.60). The increase in classification accuracy with respect to decreasing range of RSDE measures was observed from the trendlines (Figure 7). For example, JM-SAM (TAN \& SIN) measures classify Excocaria at an accuracy of $95 \%$ and $93.5 \%$ respectively. The RSDE value related to this increased classification accuracy was 2.08 and 2.07 respectively. On the other hand, SAM and JM measures yielded $75 \%$ and $77.3 \%$ at RSDE of 2.36 and 2.33 respectively.

The JM-SAM spectral matching measures resulted inan improved classification accuracy compared to the minimum distance approach. The order of classification accuracy is as follows: JM-SAM $>$ JM $>$ SAM $>$ Minimum Distance approach (Table 4). 


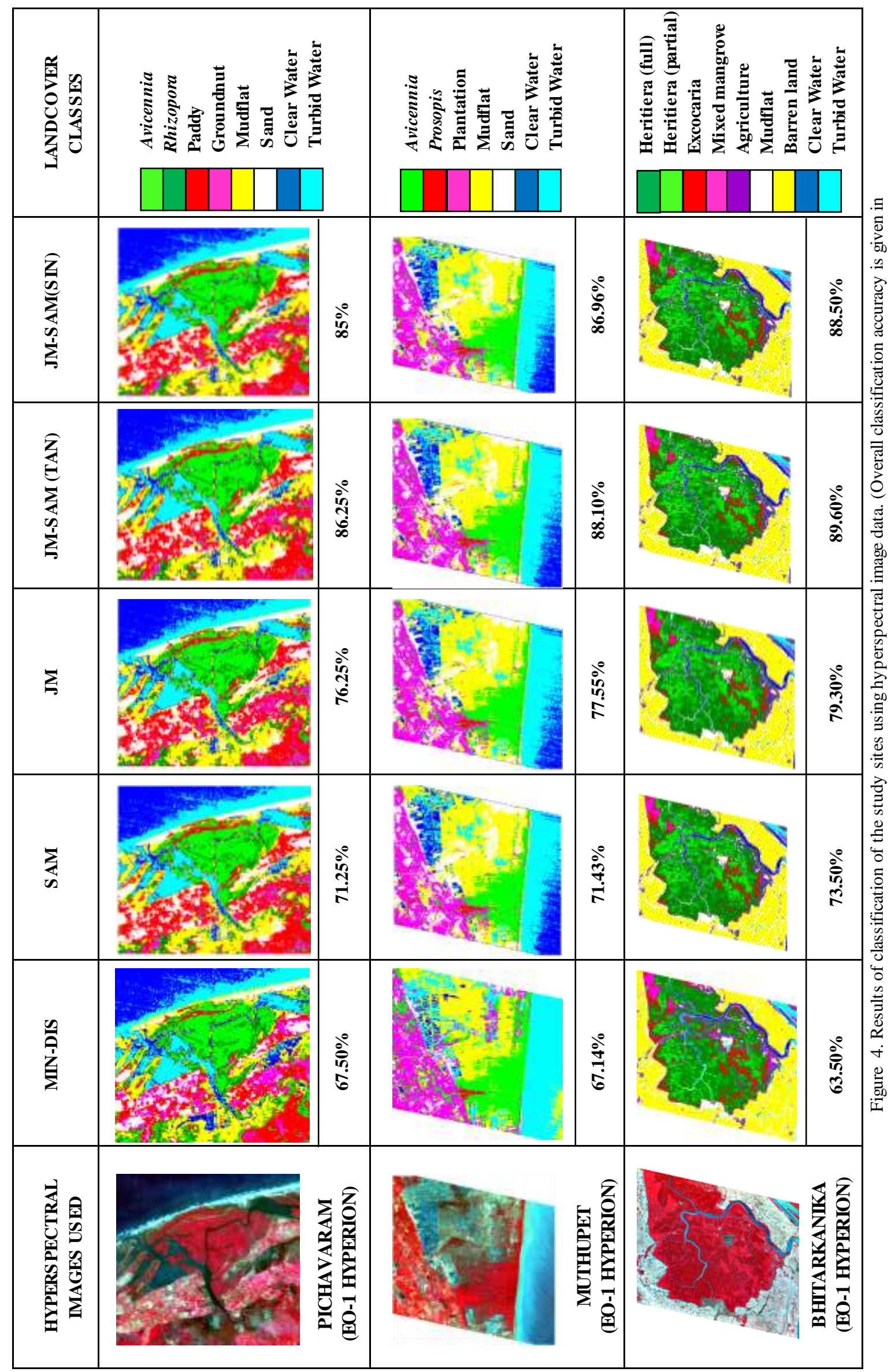




\begin{tabular}{|c|c|c|c|c|c|}
\hline \multirow{2}{*}{ Class } & \multicolumn{5}{|c|}{ Accuracy of classification (in \%) } \\
\cline { 2 - 6 } & \multirow{2}{*}{ MIN } & S AM & \multirow{2}{*}{ JM } & \multicolumn{2}{|c|}{ JM-S AM } \\
\cline { 4 - 6 } & & & & TAN & S IN \\
\hline$H F$ & 61.3 & 80 & 85 & 94 & 92.5 \\
\hline$H P$ & 59.1 & 74 & 83.3 & 93.5 & 93.1 \\
\hline$E$ & 60.1 & 75.2 & 80.10 & 85.4 & 83.5 \\
\hline$E A$ & 55.5 & 74.3 & 80.1 & 90.3 & 91.5 \\
\hline A & 40.2 & 75.5 & 82.3 & 89.5 & 88.3 \\
\hline M & 70.1 & 75.4 & 77.3 & 92.3 & 92.5 \\
\hline B & 70.6 & 76.3 & 78.3 & 89.5 & 91.1 \\
\hline C & 75.3 & 90.4 & 95.4 & 98 & 97.5 \\
\hline T & 72.4 & 91.1 & 92.5 & 98.5 & 100 \\
\hline OA & 63.5 & 73.5 & 79.3 & 89.6 & 88.5 \\
\hline OK & 0.062 & 0.70 & 0.75 & 0.85 & 0.84 \\
\hline
\end{tabular}

Note: HF-Heritiera (fully grown), HP-Heritiera (partially grown), E-Excocaria, EA-Mixed mangrove, A-Agriculture, M-Mudflat, B-Barren land, C-Clear water, T-Turbid water, OA-Overall Accuracy, OK-Overall Kappa

Table 4. Results of Accuracy Assessment for classification of Bhitarkanika image

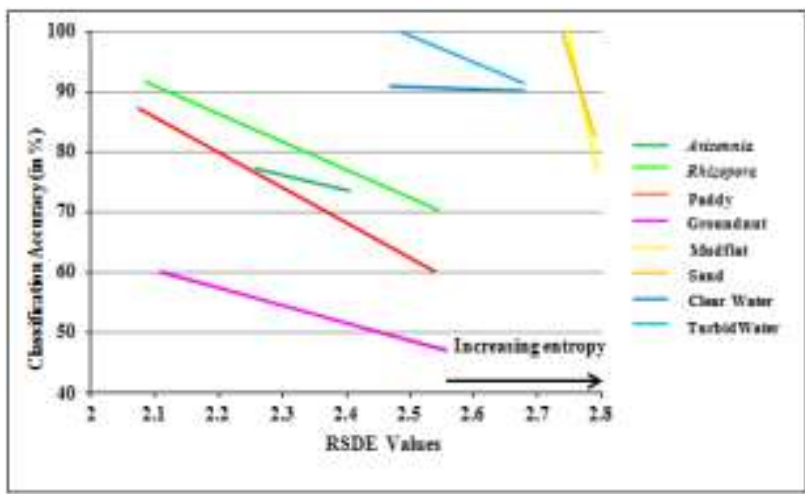

Figure 5. Trendlines depicting the relationship between the RSDE and classification accuracy for the landcover types in Pichavaram region using EO-1 Hyperion image

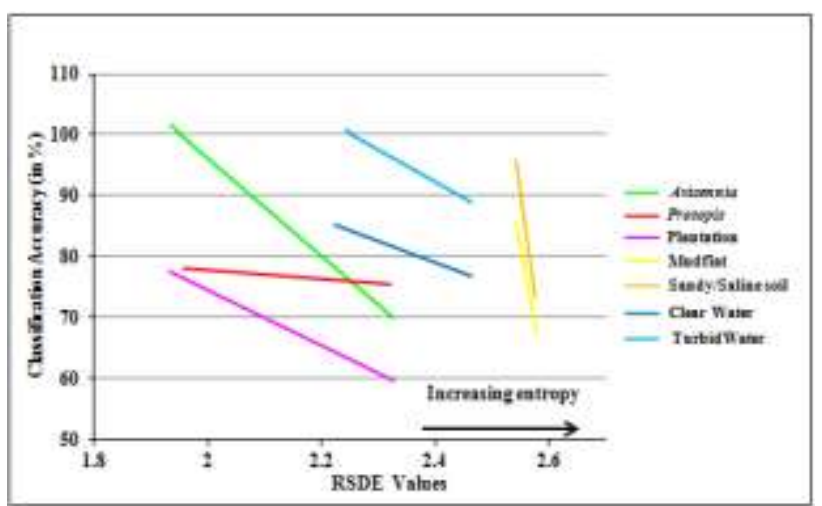

Figure 6. Trendlines depicting the relationship between the RSDE and classification accuracy for the landcover types in Muthupet region using EO-1 Hyperion image

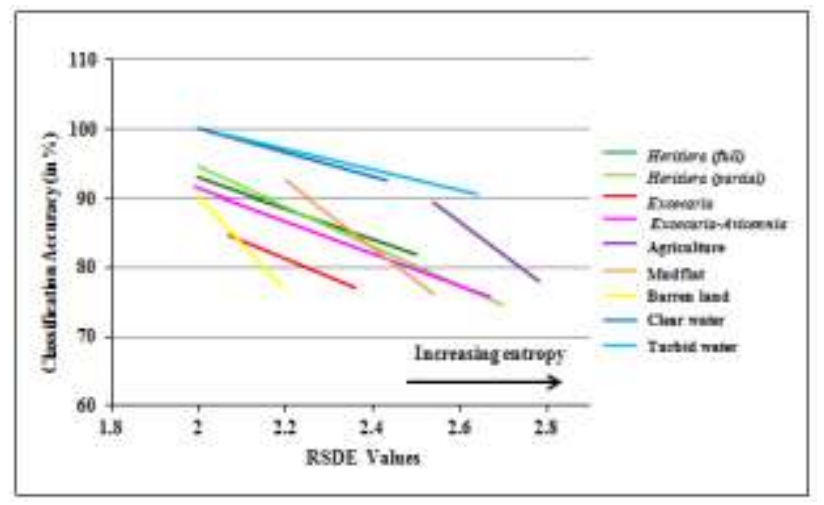

Figure 7. Trendlines depicting the relationship between the RSDE and classification accuracy for the landcover types in Bhitarkanika region using EO-1 Hyperion image

\section{CONCLUSIONS}

This paper has presented a new spectral matching measure named the 'JM-SAM approach' which combines the capabilities of the Spectral Angle Mapper (SAM) and the Jeffries-Matusita (JM) distance and proves to be effective in landcover classification. When used in image classification of the Pichavaram, Muthupet and Bhitarkanika mangrove ecosystems, the combined measures of JM-SAM (TAN) and JM-SAM(SIN) yielded an increased accuracy of $(86.25 \%, 85 \%)$, $(88.1 \%$, $86.96 \%)$ and $(89.6 \%, 88.5 \%)$ compared to the individual SAM and JM measures. Further, the spectral matching approaches namely the combined JM-SAM measures yielded a higher classification accuracy compared to the results of minimumdistance-mean classifier at an average difference of $(20.19 \%$, $20.98 \%),(19.11 \%, 17.86 \%)$ and $(26.1 \%, 25.0 \%) \%$ for all the three sites. Hence, the proposed algorithm proved its utility in extracting landcover information more accurately for a complex mangrove ecosystem through this experiment.

The simultaneous utilization of band-wise information (JM) and the geometrical aspects (SAM) of the spectrum has resulted in the efficient JM-SAM spectral matching algorithm. Besides, the compatibility of the algorithm in dealing with hyperspectral data and resulting in consistent performances in characterizing all the three study sites (Pichavaram, Muthupet, and Bhitarkanika) is observed, thus implying the applicability of the proposed algorithm to characterize other similar mangrove ecosy stems.

\section{REFERENCES}

Ajithkumar, T.T., Thangaradjou, T., Kannan, L., 2008. Spectral reflectance properties of mangrove species of the Muthupettai mangrove environment, Tamil Nadu. In: Journal of Environmental Biology, Vol. 29, no. 5, 785-788.

Beck, R., 2003. EO-1 User Guide. Version 2.3, University of Cincinnati. 
Chang, C.I., (Ed.) 2003. Hyperspectral imaging: techniques for spectral detection and classification, Kluwer Academic, New York.

Du, Y., Chang., C.I., Ren, H., Chang, C.C., Jensen, J.O., D’Amico, F.M., 2004. New hyperspectral discrimination measure for spectral characterization. In: Optical Engineering, Vol. 43, no. 8, pp. 1777-1786.

Dudeni, N., Debba, P., 2009. Evaluation of discrimination measures to characterize spectrally similar leaves of African Savannah Trees. In: 57th Biennial Session of the International Statistical Institute, 16 - 22, August 2009, South Africa.

Ghiy amat, A., Shafri, H.Z.M., Amouzad Mahdiraji, G., Shariff, A.R.M., Mansor, S., 2013. Hy perspectral discrimination of tree species with different classifications using single-and multipleendmember. In: International Journal of Applied Earth Observation and Geoinformation, Vol. 23, pp. 177-191.

Kruse, F.A., Lefkoff, A.B., Boardman, J.W., Heidebrecht, K.B., Shapiro, A.T., Barloon, P.J., Goetz, A.F.H., 1993. The spectral image processing system (SIPS) - interactive visualization and analysis of imaging spectrometer data. In: Remote Sensing of Environment, Vol. 44, no. 2-3, pp. 145-163.

Naresh Kumar, M., Seshasai, V.R., Vara Prasad, K.S., Kamala, V., Ramana, K.V., Dwivedi, R.S., Roy, P.S., 2011. A new hybrid spectral similarity measure for discrimination among Vigna species. In: International Journal of Remote Sensing, Vol. 32, no. 14, pp. 4041-4053.

Reddy, C.S., Pattanaik, C., Murthy, E.N., Raju, V.S., 2008. Community zonation of mangroves in Bhitarkanika wildlife sanctuary, Orissa, India using IRS P6 LISS III Data. In: Proceeding of the National Academy of Science-Biological Sciences, Vol. 78, no. 3, pp. 246-252.

Selvam, V., Gnanappazham, L., Navamuniyammal, M., Ravichandran, K.K., Karunagaran, V.M., 2002. Atlas of Mangrove Wetlands of India: Part 1-Tamil Nadu. M.S. Swaminathan Research Foundation, India.

Selvam, V., Ravichandran, K.K., Gnanappazham, L., Navamuniy ammal, M., 2003. Assessment of community-based restoration of Pichavaram mangrove wetland using remote sensing data. In: Current Science, Vol. 85, no. 6, pp. 794-798.

Selvam, V., Ravichandaran, K.K., Karunakaran, V.M., Mani, K.G., Evanjalin, J.B., Gnanappazham, L., 2010. Pichavaram mangrove wetlands: Situation Analysis, Chennai. M.S. Swaminathan Research Foundation, India.

Swain, P.H., King, R.C., 1973. Two effective feature selection criteria for multispectral remote sensing. LARS Technical Reports, Purdue University.
Vishnu, S., Nidamanuri, R.R., Bremananth, R., 2013. Spectral material mapping using hyperspectral imagery: a review of spectral matching and library search methods. In: Geocarto International, Vol. 28, no. 2, pp. 171-190. 